\title{
Pathologic examination of the placenta: recommended versus observed practice in a university hospital
}

This article was published in the following Dove Press journal:

International Journal of Women's Health

II June 2013

Number of times this article has been viewed

\author{
Amber Sills' \\ Carmen Steigman ${ }^{2}$ \\ Songthip T Ounpraseuth ${ }^{3}$ \\ Imelda Odibo' \\ Adam T Sandlin' \\ Everett F Magann' \\ 'Departments of Obstetrics and \\ Gynecology, University of Arkansas \\ for the Medical Sciences, Little Rock, \\ AR, USA; ${ }^{2}$ Department of Pathology, \\ University of Arkansas for the \\ Medical Sciences, Little Rock, AR, \\ USA; ${ }^{3}$ Department of Biostatistics, \\ University of Arkansas for the Medical \\ Sciences, Little Rock, AR, USA
}

Correspondence: Everett F Magann Department of Obstetrics and Gynecology, University of Arkansas for the Medical Sciences, 430I W. Markham St, Slot \# 518, Little Rock, AR, USA 72205

$\mathrm{Tel}+\mathrm{I} 50 \mid 6868345$

$\mathrm{Fax}+\mathrm{I} 5015267820$

Email efmagann@uams.edu
Introduction: The purpose of this study was to determine the frequency of appropriate placental examinations in a university hospital.

Methods: A retrospective review of all deliveries and all placentas submitted for pathologic examination from live births. Placentas were reviewed by a perinatal pathologist to determine whether they met the College of American Pathologists (CAP)-recommended guidelines for examination.

Results: We used 1346 deliveries between July 1, 2010 and December 31, 2010 as the basis of this review. According to CAP guidelines, 703 placentas (52.2\%) should have been sent for pathologic examination; $575 / 703(81.8 \%$; 95\% confidence interval $[\mathrm{CI}]=78.9-84.7)$ were actually sent for examination. Of the 643 placentas that did not need to be examined per CAP guidelines, 568 $(88.3 \% ; 95 \% \mathrm{CI}=85.9-90.8)$ were appropriately not sent. In comparing the three categories of indications for examination (maternal, fetal/neonatal, placental), the only significant association was that women with fetal/neonatal indications were more likely to have their placenta sent than women with maternal indications (odds ratio, 2.63; 95\% CI $=1.81-3.80$ ).

Conclusion: In this university hospital, more than $80 \%$ of the time, placentas were appropriately sent to pathology, and more than $85 \%$ of the time, placentas that should not have been sent for evaluation were not sent.

Keywords: placenta, pathologic examination, clinical guidelines, birth

\section{Introduction}

The gross and microscopic examination of the placenta is recommended and considered to be an essential part of the evaluation following any stillbirth. ${ }^{1}$ However, recommendations about which placentas should be sent for pathologic examination following the vaginal or cesarean delivery of a live-born fetus remain unclear. In a 1993 publication by the American Congress of Obstetricians and Gynecologists (ACOG) on placental examination, no specific recommendations were given. ${ }^{2}$ In a recent assessment of both the committee opinions and practice bulletins from ACOG, the 1993 committee opinion was no longer present on the website and no new recommendations about which placentas should be examined could be found. ${ }^{3}$ In a search of resources and publications on the websites of both the Royal Australian and New Zealand College of Obstetricians and Gynaecologists (RANZCOG) and the Royal College of Obstetricians and Gynaecologists (RCOG) in the United Kingdom, ${ }^{4,5}$ no recommendations could be found on placental examination following a live birth.

In 1997, the College of American Pathologists (CAP) published guidelines for placental pathological examination following delivery. ${ }^{6}$ The recommendations were for 
underlying maternal disease, pregnancy complications, fetal/ neonatal conditions, or placental indications. These guidelines have been adopted at many academic centers; however, many practicing obstetricians and labor and delivery nursing staff are not aware of the specific CAP guidelines for pathological evaluation of the placenta. Several studies have shown a very low sensitivity but high specificity in the likelihood of placental examinations based on CAP guidelines. ${ }^{7,8}$

The primary purpose of this study was to determine the frequency of appropriate placental pathological examinations in a university hospital. The actual indications for which placental pathological examinations were being performed in a university setting and whether they met criteria set in place by the CAP guidelines were evaluated as well. Additionally, the frequency of no examination on noneligible placentas also was analyzed.

\section{Methods}

This is a retrospective review of all obstetrical deliveries that resulted in a live birth and all placentas submitted for pathologic examination at the University of Arkansas for Medical Sciences (UAMS) between July 1, 2010 and December 31, 2010. This study was approved under expedited review by the UAMS Institutional Review Board (number 133217).

Obstetrical records from the delivery logs and the electronic medical records were reviewed for the clinical birth history on each obstetrical delivery case, including whether or not the placenta was submitted to pathology for examination. Information was obtained from all placental requisitions and placental diagnostic reports generated by the Department of Pathology at UAMS during that same time interval. The pathology requisitions and the pathology reports from all of the submitted placentas were then reviewed by a single perinatal pathologist to determine whether the placentas met the CAP-recommended guidelines for pathologic examination. The CAP guidelines for placental pathological examination encompass three categories: (1) maternal indications, (2) fetal/neonatal indications, and (3) placental indications. The maternal indications include systemic disorders, preterm delivery $\leq 34$ weeks, peripartum fever/infection, unexplained third-trimester bleeding, clinical concern for infection, severe oligohydramnios, unexplained or recurrent pregnancy complication, abruption, invasive procedure with suspected placental injury, nonelective pregnancy termination, and thick meconium. Fetal/neonatal indications include infant admitted to other than a level-one nursery, stillbirth/perinatal death, compromised clinical condition, hydrops fetalis, birth weight less than the tenth percentile, seizures, infection or sepsis, major anomalies, discordant twin growth, and multiple gestations with same-sex twins. Placental indications include physical abnormalities (infarct, mass, vascular thrombosis, retroplacental hematoma, amnion nodosum, abnormal coloration or opacification, malodor), small or large placenta, umbilical cord lesion, and cord length $<32 \mathrm{~cm}$.

Comparisons were made between guidelines for placental pathological studies and actual indications for examination. Each case was categorized as a true or false positive on the basis of the CAP guidelines for placental examination. Sensitivity, specificity, and prevalence with $95 \%$ confidence intervals (CI) were calculated. Additionally, descriptive analyses of key demographic data using independent $t$-tests and Chi-square tests, as appropriate, were used to compare patients whose placental pathological evaluations were verified with those whose evaluations were not verified. To help identify which CAP indicators predict placental examination, the odds ratios (ORs) and 95\% CIs for placental examination based on the individual CAP clinically recommended indications were calculated. Finally, the patients were divided into four categories on the basis of the CAP guidelines: category 0 had no identifiable maternal, fetal/neonatal, or placental indications; category 1 had maternal indications; category 2 had fetal/neonatal indications; and category 3 had placental indications. These categories were examined for significant differences between the individual categories and the likelihood that the placenta was sent for examination. All statistical analyses were performed using SAS 9.2 (SAS Institute, Inc, Cary, NC, USA) with $P$-values $<0.05$ considered statistically significant.

\section{Results}

A total of 1347 deliveries were performed between July 1, 2010 and December 31, 2010 at UAMS. Of these, 1346 were correctly identified by medical record number and are the basis of this review. A total of 575 placentas were sent for pathological evaluation. Review of the records by a perinatal pathologist following the CAP guidelines revealed that 703 placentas $(52.2 \%)$ should have been sent for pathologic examination. Among these placentas, 575/703 (81.8\%; 95\% CI = 78.9-84.7) were actually sent for pathologic examinations. Of the 643 placentas ascertained by the perinatal pathologist as not needing to be sent for pathological examination, 568 (88.3\%; 95\% CI = 85.9-90.8) were appropriately not sent (Table 1 ). The level of agreement as to whether a placenta should or should not have been sent for pathological examination among the practicing obstetricians and/or labor and delivery nursing staff, compared 
Table I Correctness of sending the placenta for pathologic examination

\begin{tabular}{llll}
\hline Was it sent? & \multicolumn{3}{l}{ Should it be sent? } \\
\cline { 2 - 4 } & Yes & No & \\
\hline Yes & $575^{*}$ & 75 & $\begin{array}{l}\text { Positive predictive value: } \\
88.4 \%\end{array}$ \\
No & 128 & $568^{*}$ & $\begin{array}{l}\text { Negative predictive value: } \\
\end{array}$ \\
& & & $81.6 \%$ \\
& Sensitivity: & Specificity: & \\
& $81.8 \%$ & $88.3 \%$ & $\kappa=0.70$
\end{tabular}

Note: *Placentas that were appropriately handled per College of American Pathologists guidelines.

with the review by the perinatal pathologist following the CAP guidelines, was substantial, with a kappa coefficient $(\kappa)=0.70(95 \% \mathrm{CI}=0.66-0.74)$.

An overall analysis by CAP indications $(0=$ none identified, $1=$ maternal, $2=$ fetal $/$ neonatal, $3=$ placental $)$ revealed a significant association between the indication categories and whether or not the placenta was sent for examination $(P<0.0001)$ (Table 2). Further analysis revealed a significant likelihood of the placenta being sent for maternal indications $(\mathrm{OR}=5.86 ; 95 \% \mathrm{CI}=4.31-7.96)$, fetal/neonatal indications $(\mathrm{OR}=15.38 ; 95 \% \mathrm{CI}=9.94-23.81)$, and placental indications $(\mathrm{OR}=9.95 ; 95 \% \mathrm{CI}=3.17-29.01)$ compared with the category without identifiable indications. In comparing the three categories with indications (maternal, fetal/ neonatal, placental) with whether or not the placenta was sent for examination, the only significant association was that women with fetal/neonatal indications were more likely than the women with maternal indications to have their placentas sent $(\mathrm{OR}=2.63 ; 95 \% \mathrm{CI}=1.81-3.80)$.

\section{Discussion}

Potential benefits of pathological examination of the placenta include the evaluation and explanation of the etiology associated with an adverse pregnancy outcome, the formulation of a plan of management for future pregnancies, the capability to predict the risk for long-term neonatal neurodevelopmental

Table 2 Association between College of American Pathologists indications and whether or not the placenta was sent for examination

\begin{tabular}{lll}
\hline Indication & Sent, N (\%) & \\
\cline { 2 - 3 } & No & Yes \\
\hline 0. None & $302(83 \%)$ & $63(17 \%)$ \\
I. Maternal & $346(45 \%)$ & $423(55 \%)$ \\
2. Fetal/neonatal & $43(24 \%)$ & $138(76 \%)$ \\
3. Placental & $5(33 \%)$ & $10(67 \%)$ \\
\hline
\end{tabular}

Abbreviation: $\mathrm{N}$, number. problems, and medical-legal risk assessment of an adverse pregnancy outcome. ${ }^{9}$ This has led some experts to suggest that placental pathology should be a routine component of obstetric and neonatal care. ${ }^{10}$ However, there is confusion regarding these potential benefits of placental examination; this has been largely attributed to the inadequate training of both obstetricians and placental pathologists. ${ }^{7-11}$ This is further compounded by the lack of guidelines by ACOG, ANZCOG, and RCOG for placental examination. ${ }^{3-5}$

In our study, over a 6-month period of time in 2010, 48.3\% of placentas (575 correctly and 75 incorrectly) were sent to pathology. Among all deliveries, $52.2 \%$ had indications for pathologic examination of the placenta on the basis of the 1997 CAP guidelines. Of these, placental examination was undertaken in $81.8 \%$. Among the placentas that did not need pathologic examination per the CAP guidelines, $88.3 \%$ were appropriately not sent and $11.7 \%$ were sent and examined. These findings differ from CAP guideline compliance evaluations undertaken at other universities 5-10 years earlier. In a South Australian study of deliveries that occurred over the course of 3 months in 2000, 49.5\% of all deliveries met CAP guidelines for placental examination; however, placental examination occurred in only $17.8 \%$ of these deliveries. ${ }^{7}$ Only $1.1 \%$ of placentas not meeting CAP guidelines were examined. In a US investigation, Curtin et $\mathrm{al}^{8}$ reviewed more than 3000 deliveries over the same time period in 2001 and found that $37.5 \%$ of all deliveries qualified for pathologic examination of the placenta, but only $18.2 \%$ had a placental examination. Of all submitted placentas, 93\% had appropriate CAP indications. In 2007, Al Harazi and Frass ${ }^{11}$ reviewed the records of more than 11,000 deliveries in Yemen and found that although 13\% met CAP guidelines for placental examination, only $4.9 \%$ were actually examined. In all of these studies, oversubmission of placentas for examination was not an issue; rather, there was undersubmission according to the CAP guidelines.

The reason for the apparent increase in the frequency of appropriate submissions in our study is unclear. A previous study attributed low submission rates to lack of awareness of the need for examination, lack of clear guidelines, lack of communication between obstetricians and pathologists, and lack of understanding of unfamiliar histologic terms on the part of the obstetrician that leads to failure to use the information provided. ${ }^{11}$ Our institution has adopted the CAP guidelines for pathological examination of the placenta. At every delivery, as part of the delivery protocol by the nursing staff, the delivering health care provider is asked if the placenta should be disposed of or sent for pathologic examination. 
It is uncertain if the practicing obstetricians and residents are aware of these specific guidelines; however, they are asked to make a decision after every delivery as to whether the placenta should be sent for examination or not. This has resulted in a higher percentage of placentas being correctly sent (or not sent) for examination.

A review of indications revealed that women with fetal/ neonatal indications were significantly more likely than those with maternal indications to have their placentas sent for examination. Other studies have shown high rates of placentas being sent for examination if the pregnancies were shorter than 34 weeks or if the woman had a multiple gestation. ${ }^{7,8}$ This suggests that obstetricians are in agreement and more likely to recognize fetal/neonatal indications as a valid indication to send the placenta.

One of the limitations of this study was that we had only a single pathologist review the appropriateness of sending or not sending the placenta for examination. A review by more than one pathologist would have been helpful; however, there is only one perinatal pathologist at our university and, in fact, in the entire state of Arkansas.

Finally, although cost effectiveness is an important issue in health care today, the importance of appropriate placental examination and its benefits cannot be over emphasized. Compared with the price for a single malpractice settlement related to cerebral palsy or other disabilities, the charges for placenta examination are minuscule. ${ }^{10}$ On the basis of the studies reviewed, $13 \%-50 \%$ of placentas will require pathologic exam according to CAP guidelines. This is a considerable number; therefore, familiarization with the guidelines should be undertaken to further reduce the cost of unnecessary examinations. Each institution should strive to adopt the CAP guidelines or have its own set of guidelines based on available scientific evidence. Most importantly, the indications should be communicated to the staff.

\section{Conclusion}

Vital information can be obtained from placental pathological examination, including insight to the underlying disease process(es) influencing the antenatal course and perinatal outcome. Hence, obstetricians should strive to better utilize this information in order to improve maternal and neonatal outcomes.

\section{Acknowledgments}

Special thanks to Donna Eastham, BA, for her editorial work on this manuscript.

\section{Disclosure}

The authors report no conflicts of interest in this work.

\section{References}

1. American Congress of Obstetricians and Gynecologists. Management of stillbirth. ACOG Practice Bulletin 102. Washington, DC: American Congress of Obestricians and Gynecologists; 2009:102(383).

2. Placental pathology. ACOG Committee Opinion: Committee on Obsetrics: Maternal and Fetal Medicine. Number 125-Jul 1993. Int $J$ Gynaecol Obstet. 1993;42(3):318-319.

3. The American Congress of Obstetricians and Gynecologists. Resources and publications [webpage on the Internet]. Washington, DC; American Congress of Obstetricians and Gynecologists; 2013. Available from: http://www.acog.org/Resources_And_Publications. Accessed September 6, 2012.

4. The Royal Australian and New Zealand College of Obstetricians and Gynaecologists. College statements and guidelines [homepage on the Internet]. Victoria, Australia: The Royal Australian and New Zealand College of Obstetricians and Gynaecologists; 2011 [updated October 19, 2012]. Available from: http://www.ranzcog.edu.au/theranzcog/policies-and-guidelines/college-statements.html. Accessed September 6, 2012.

5. Royal College of Obstetricians and Gynaecologists. RCOG guidelines [webpage on the Internet]. London, UK: Royal College of Obstetricians and Gynaecologists; 2013. Available from: http://www.rcog.org.uk/ guidelines. Accessed September 6, 2012.

6. Langston C, Kaplan C, Macpherson T, et al. Practice guideline for examination of the placenta: developed by the Placental Pathology Practice Guideline Development Task Force of the College of American Pathologists. Arch Pathol Lab Med. 1997;121(5):449-476.

7. Spencer MK, Khong TY. Conformity to guidelines for pathologic examination of the placenta. Arch Pathol Lab Med. 2003;127(2):205-207.

8. Curtin WM, Krauss S, Metlay LA, Katzman PJ. Pathologic examination of the placenta and observed practice. Obstet Gynecol. 2007:109(1): $35-41$.

9. Benirschke K. The placenta in the litigation process. Am J Obstet Gynecol. 1990;162(6):1445-1448; discussion 1448-1450.

10. Salafia CM, Vintzileos AM. Why all placentas should be examined by a pathologist in 1990. Am J Obstet Gynecol. 1990;163(4 Pt 1): 1282-1293.

11. Al Harazi AH, Frass KA. Low rate of placenta pathological examination in a tertiary care hospital in Sana'a, Yemen. East Mediterr Health J. 2011;17(4):277-280.
International Journal of Women's Health

\section{Publish your work in this journal}

The International Journal of Women's Health is an international, peerreviewed open-access journal publishing original research, reports, editorials, reviews and commentaries on all aspects of women's healthcare including gynecology, obstetrics, and breast cancer. The manuscript management system is completely online and includes

\section{Dovepress}

a very quick and fair peer-review system, which is all easy to use. Visit http://www.dovepress.com/testimonials.php to read real quotes from published authors. 